\title{
Stuck State Avoidance in Work Vehicle's Control with Hybrid Twin Framework
}

\author{
Atsuhiro Aoki ${ }^{1}$, Hideaki Shimamura ${ }^{1}$, Munetaka Nakata ${ }^{2}$, Kazuhiro Motegi ${ }^{2}$, Yoichi Shiraishi ${ }^{2, *}$ \\ ${ }^{1}$ Life Creation Center, Honda R\&D Co., ltd., Saitama, 351-0024, Japan \\ ${ }^{2}$ Department of Mechanical Science and Technology, Gunma University, Gunma, 373-0057, Japan
}

Received February 21, 2020; Revised April 17, 2020; Accepted May 3, 2020

Copyright $\odot 2020$ by authors, all rights reserved. Authors agree that this article remains permanently open access under the terms of the Creative Commons Attribution License 4.0 International License

\begin{abstract}
This paper suggests an IoT device utilization for skid suppression for work vehicles in the framework of Hybrid Twin and the performances of IoT utilization are experimentally evaluated. In the autonomous driving of work vehicles, stuck state or lock state should be avoided because it is very difficult to escape from these states autonomously. First of all, a state transition diagram consisting of grip, skid, stuck and lock states is configured and a skid ratio is newly defined to measure the degree of skid. Then, a three-stage skid suppression method is suggested based on skid ratio and a controlling system is implemented by using MATLAB/Simulink. Using an actual snow blower, an IoT device with some filtering processes is incorporated and the three-stage skid suppression method is applied. The experimental results show that the suggested approach is feasible but not a three-stage but a two-stage skid suppression method is likely to be efficient because of insufficient noise reduction. As future work, the noise reduction should be improved and the skid suppression method should be implemented in a Hybrid Twin approach.
\end{abstract}

Keywords IoT, Skid, Sensor, Model Based Design, Digital Twin

\section{Introduction}

These days, such work vehicles as maintenance vehicles, bulldozers, grass cutters, and snow blowers are gradually being motorized. The electric system with motors is adequate for detailed control and it is strongly required that these work vehicles should be carrying autonomous driving function [1]. Different from passenger vehicles, the work vehicles must drive on wasteland, muddy ground, or ground covered with snow, etc. Here, the vehicle's power train should recover from a skid state automatically even if it falls into such state, because the skid state may proceed to stuck or lock state if no control is executed.

Once a work vehicle is in these states, it is impossible to return to grip state by itself. As an example of snow blower, when it remains in a skid state, the snow under the crawler is dug out and the bottom of body touches the snow surface and as a result, the snow blower cannot move any more. This is defined a stuck state.

The detailed analysis on the mechanism of rubber crawler has already been reported [2] but this is on the inner structure of crawler and no discussions on skid, stuck and lock states are found. The modeling of hydraulic excavator with crawlers has been reported [3], but this is on the vibration analysis of crawler and body. In MathWorks, the anti-lock braking system is modeled dynamically [4] and the modeling of skid phenomenon is useful. However, here, the focus is just on the braking system.

This paper first configures the state transition diagram consisting of grip, skid, stuck, and locks states and analyzes the state transition conditions accurately. Next, a skid ratio newly defined by using the body speed and the drive-train speed to measure the degree of skid. The skid ratio is measured by using an IoT device attached to the work vehicle. Then, based on the skid ratio, a three-stage skid suppression method is suggested to carefully get the work vehicle back to grip state from skid state so as not to go into stuck or lock state.

The Model Based Design (MBD) method is indispensable for designing controllers in an automobile development [5] and in many other systems. The MBD method is already applied to the design of electric vehicles and attained efficient results [6, 7] and this method is applied to solve the problems dealt with in this paper. The MBD method is effective for only designing control systems and the Hybrid Twin ${ }^{\mathrm{TM}}$ [8] is developed for operating completed control systems dynamically and optimally. This paper suggests the utilization of acceleration sensor for detecting the skid state as an IoT 
device which maps the driving state of work vehicle in the real system into the corresponding state as a Digital Twin. The obtained acceleration signal has much noise and three kinds of filters are applied for noise reduction. In the experiments with snow blower, the skid state is detected by estimating the skid ratio and not a three-stage but a two-stage skid suppression method based on the skid ratio is applied because of insufficient noise reduction. However, the two-stage skid suppression method itself would have a promising performance in the practical application.

In the followings, the problems dealt with in this paper are defined and the Hybrid Twin approach is described in Chapter 2. Chapter 3 is dedicated to the modeling of driving states of work vehicle in the Virtual Twin. The model of work vehicle's controller is implemented by MATLAB/Simulink and three-stage skid suppression method is shown in Chapter 4. Chapter 5 is assigned to the experimental results with a snow blower. Here, three kinds of filters for obtaining skid ratio from actual acceleration sensor signal are shown and two-stage skid suppression method is suggested. Finally, this paper is concluded in Chapter 6.

\section{Work Vehicle Control and Hybrid Twin Framework}

\subsection{Problems in Work Vehicle's Control}

The work vehicles with crawlers must drive on wasteland, muddy ground, or ground covered with snow, etc., and they often go into the skid state. The skid state and the stuck state are shown in Fig. 1 and the vehicle should recover from a skid state automatically when the vehicle drives autonomously. Because, the skid state may proceed to stuck state shown in Fig. 1 if no control is executed. In case of a snow blower, it is defined a stuck state that the bottom of the vehicle touches the snow surface just under the vehicle because the snow under the crawler is dug out if the crawler continues to rotate. Once a work vehicle is in stuck state, it is impossible to return to normal grip state by itself. Therefore, the problems are that firstly, the skid state should be detected and secondly, the work vehicle should be controlled to return to the grip state so as not to fall into stuck state.

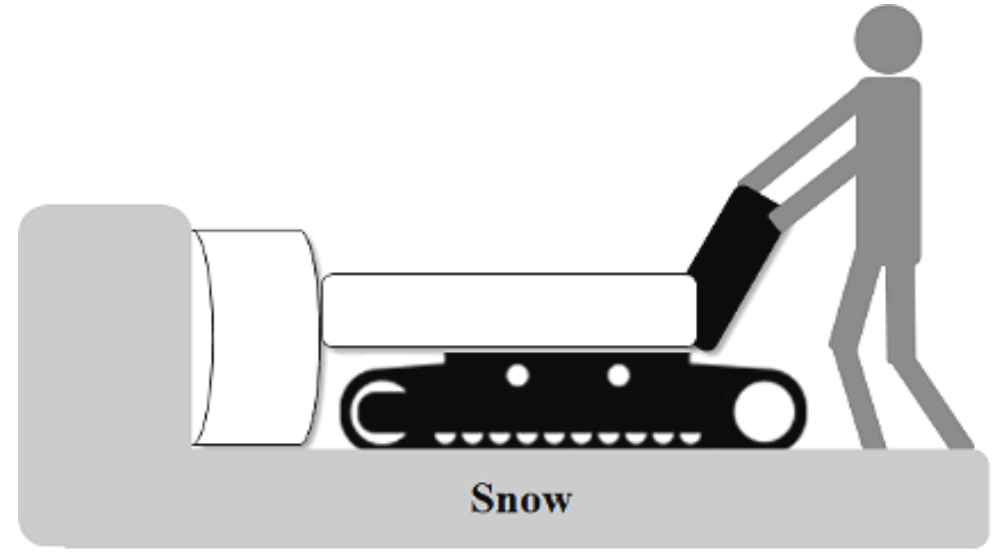

Skid State

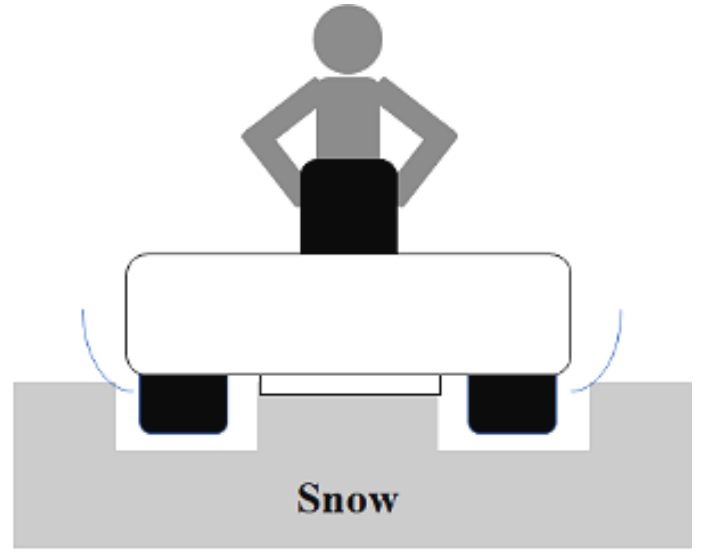

Stuck State

Figure 1. Skid and stuck states of snow blower 


\subsection{Hybrid Twin Approach}

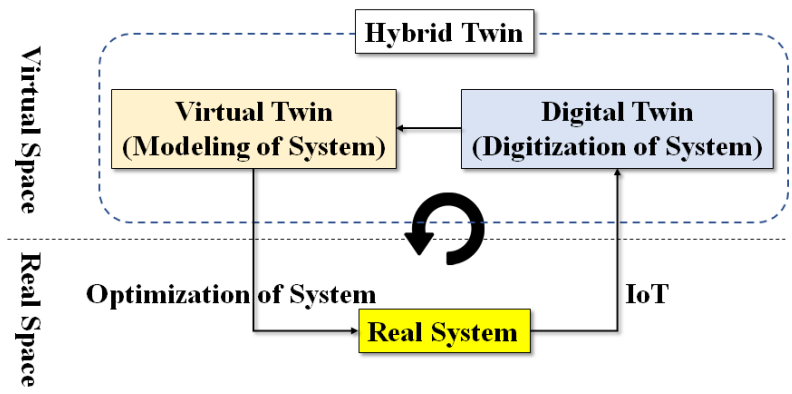

Figure 2. Digital Twin, Virtual Twin and Hybrid Twin

A Hybrid Twin ${ }^{\mathrm{TM}}[8]$ consisting of Virtual Twin and Digital Twin is used to mainly control an on-line system optimally. This approach is the extension of Digital Twin [9] and a Virtual Twin is added for separating the models for simulation from Digital Twin as shown in Fig. 2. Here, the Digital Twin is the numerical representation of real system while the Virtual Twin is the modeling of the system. The set of parameters extracted in the Digital Twin by, for example, IoT devices, is the input to the Virtual Twin. The models in the Virtual Twin are executed and as a result, the controlling parameters are given back to the real system. The loop consisting of Digital Twin, Virtual Twin and optimization of real system is repeated with the specified time step. Therefore, the Digital and the Virtual Twins must be executed very fast and a technique to convert a 3-D simulator to the corresponding 1-D simulator is required. The Hybrid Twin approach has already been applied to such mission-critical control systems as in wind-generation, nuclear power reactor or smart factories. In the followings, the Hybrid Twin approach is applied to solve the problems in work vehicle's control.

\section{Modeling of Driving States of Work Vehicle in Virtual Twin}

\subsection{Driving State Definition by Crawler Speed and Body Speed}

The driving states of work vehicle with crawlers are defined as "grip state", "skid state", "stuck state" and "lock state." These states are modeled by using the body speed $V_{b}(t)$ and the crawler speed $V_{c}(t)$ as shown in Fig. 3. The driving load $F_{l}(t)$ also affects these speeds as well as the ground condition for work vehicles.

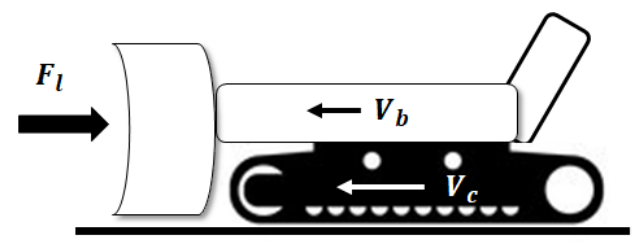

Figure 3. Body speed and crawler speed

As shown in Fig. 4, when $V_{b}=V_{c}$, here, the time parameter is omitted, the work vehicle is in a grip state. If $V_{c}$ becomes larger than $V_{b}$, the work vehicle goes into skid state or in stuck state as mentioned in Fig. 1.

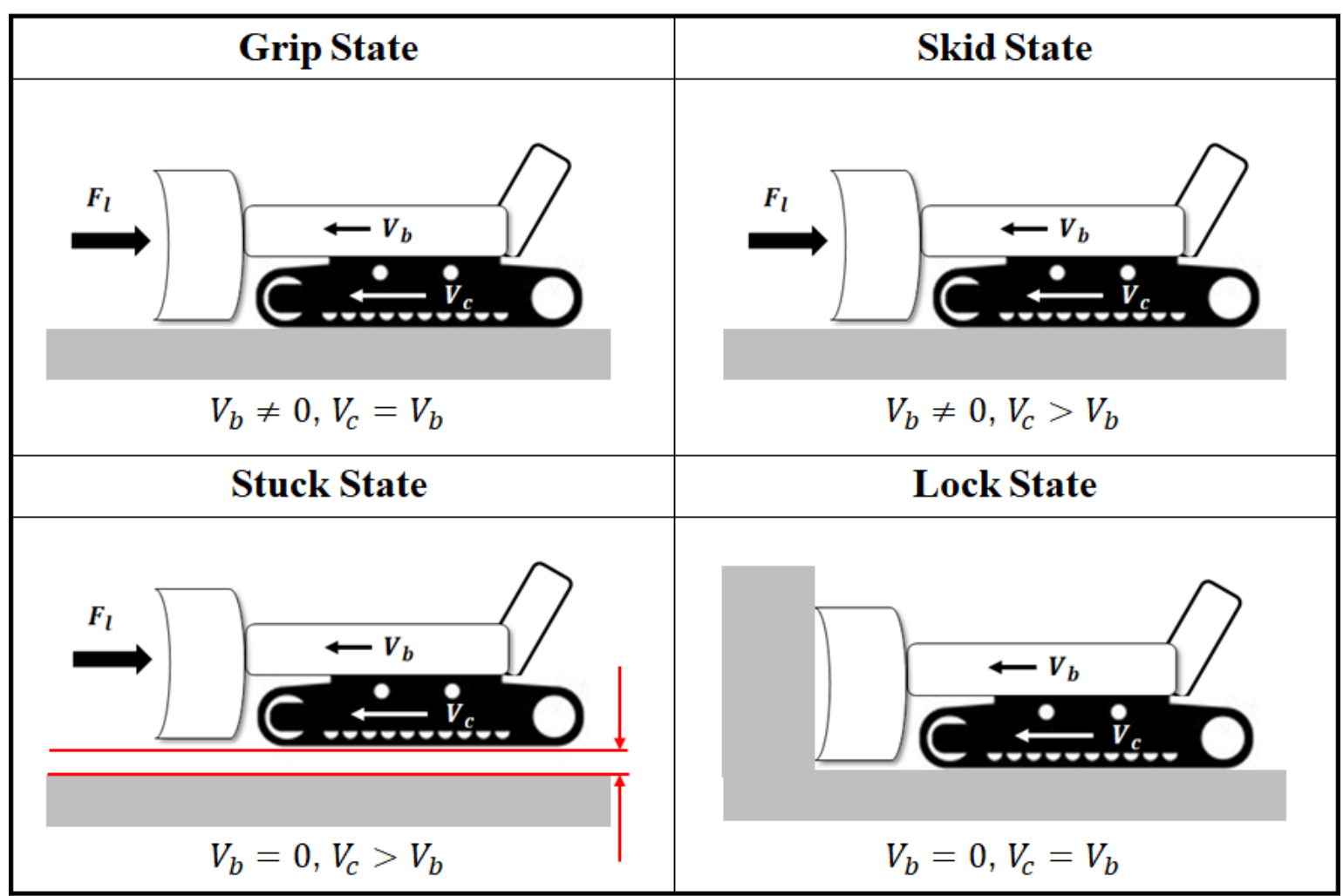

Figure 4. Work vehicle's driving states 
The difference between skid and stuck states is the body speed $V_{b}$. In the stuck state, $V_{b}=0$ as described with Fig. 1. In the lock state, the vehicle meets heavy load such as thick snow wall and the vehicle cannot move at all. The transitions among these states are shown in the state transition diagram shown in Fig. 5. Each of the states is defined by comparing $V_{b}$ and $V_{c}$ as shown in this figure. When the crawler speed $V_{c}$ in the grip state is increasing or the body speed $V_{b}$ is decreasing, the vehicle is going into the skid state. In order to get out of the skid state, $V_{c}$ must be reduced or $V_{b}$ should be increased. The skid state is the entrance to stuck state or lock state which must be avoided under the autonomous driving. Therefore, a controller to reduce the crawler speed $V_{c}$ is necessary and this is the original idea as well as utilizing the skid ratio defined next.

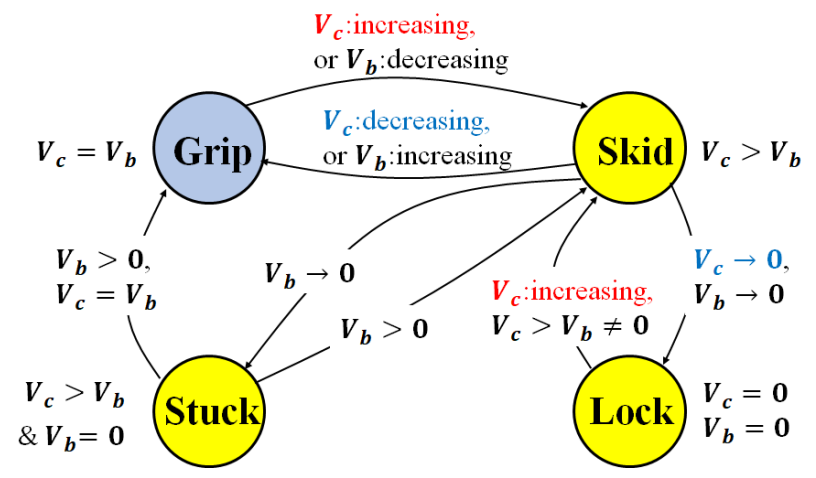

Figure 5. Sate transition diagram

\subsection{Skid Raito Estimation}

The skid ratio $\lambda$ is defined by equation (1) by using $V_{c}$ and $V_{b}$. Such a state with $\lambda=0, \lambda \rightarrow 1$ or $\lambda=1$ corresponds to grip state, skid state or stuck state, respectively, as shown in Fig. 5. The crawler speed $V_{c}$ is obtained by such physical parameters on the ground as static frictional force, dynamic frictional force, rolling resistance force, resistive load as well as driving force given to the crawler itself [7]. On the other hand, the body speed $V_{b}$ is not obtained analytically and in the previous approach [7], a dedicated wheel is used for directly measuring $V_{b}$. However, a dedicated wheel is not desirable because of its durability for a snow-covered ground or muddy ground, etc.

In case of passenger electric vehicle, Fujii and Fujimoto [10] suggested the usage of acceleration sensor for slip ratio (or skid ratio) control without any dedicated wheel. This method is likely to be applicable to the case of hybrid work vehicle with combustion engine although it has a large amount of noise and vibration. The equations for estimating slip ratio (or skid ratio) $\lambda$ are shown below.

$$
\begin{gathered}
\lambda=\frac{V_{c}-V_{b}}{V_{c}}, \\
J_{\omega} \dot{\omega}=T-r F_{l}, \\
M \dot{V}_{b}=F_{d}-F_{l}, \\
V_{c}=r \omega,
\end{gathered}
$$

$$
\dot{\hat{\lambda}}=-\frac{\dot{\omega}}{\omega} \hat{\lambda}+\left(1+\frac{J \omega}{r^{2} M}\right) \frac{\dot{\omega}}{\omega}-\frac{T}{r^{2} M \omega}+\frac{\frac{T-\dot{\omega} J \omega}{r}-(M a)}{r M \omega} .
$$

Here, $J_{\omega}, \omega, T, r, F_{l}, M, V_{b}$, and $F_{d}$ are inertia moment at rotating shaft of wheel, angular speed of motor, motor torque, wheel radius, driving load, vehicle weight, body speed and driving force, respectively. These physical parameters are shown in Fig. 6. Especially, only the parameter " $a$ ", the acceleration of vehicle, is needed for estimating the skid ratio $\lambda$ in equation (5). The estimated skid ratio $\hat{\lambda}$ is obtained by solving differential equation (5). The calculation of skid ratio $\hat{\lambda}$ corresponds to the 1-D simulator for skid state detection in Virtual Twin and the acceleration " $a$ " is the output of Digital Twin.

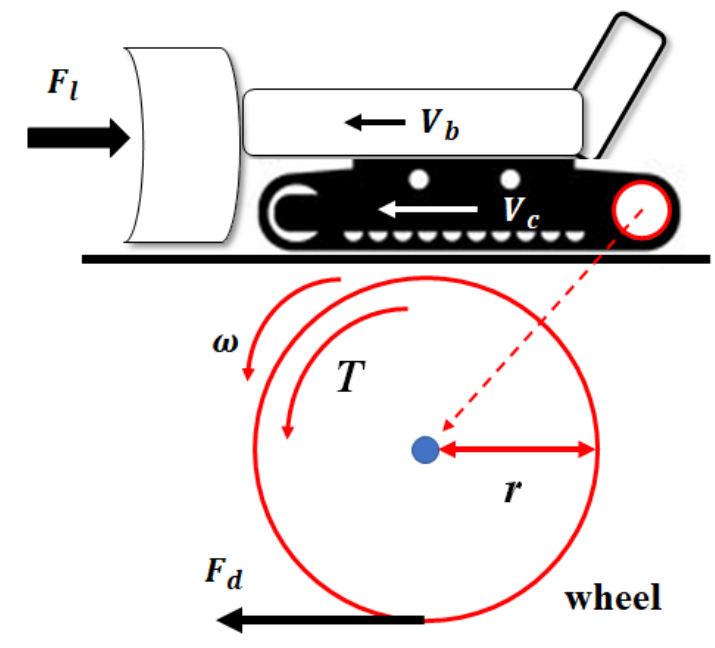

Figure 6. Crawler's physical model

\section{Modeling of Controller and Plant}

\subsection{Model Based Design Approach}

The Model Based Design (MBD) method [5] is an indispensable tool for designing and developing complicated and highly reliable embedded controller, such as ECU's (Electronic Control Units) in automobiles. In this method, the co-simulator which simulates the whole vehicle is implemented by connecting models. The MBD method has some design phases consisting of controller (ECU) and plant in real and virtual spaces. The controller and the controlled objects (plants) are modeled into virtual space. The interactions between controller and plant in the virtual space are sufficiently simulated and the controller can be efficiently designed.

\subsection{Modeling of High-Level Controller}

The top view of snow blower model is shown in Fig. 7. This model can be extended to vehicles with crawlers. The snow blower model consists of high-level controller, power system, and drive system controller. The top view of plant 
is a block named "Drive System Plant." The details of plant model are found in [11], where the interactions between environments and crawlers are precisely described. Finally, the hardware and software co-design method is applied to partition the high-level controller into hardware (micro-processor) and software (embedded program).
In Fig. 7, the target speed $V_{o}(t)$, the crawler speed $V_{c}(t+\Delta t)$ in the next time step, the estimated skid ratio $\lambda \widehat{(t)}$, the generated (necessary) torque $F_{c}(t+\Delta t)$ and the measured acceleration $a(t)$ are given to the high-level controller and it generates the next speed $V_{s}(t+\Delta t)$.

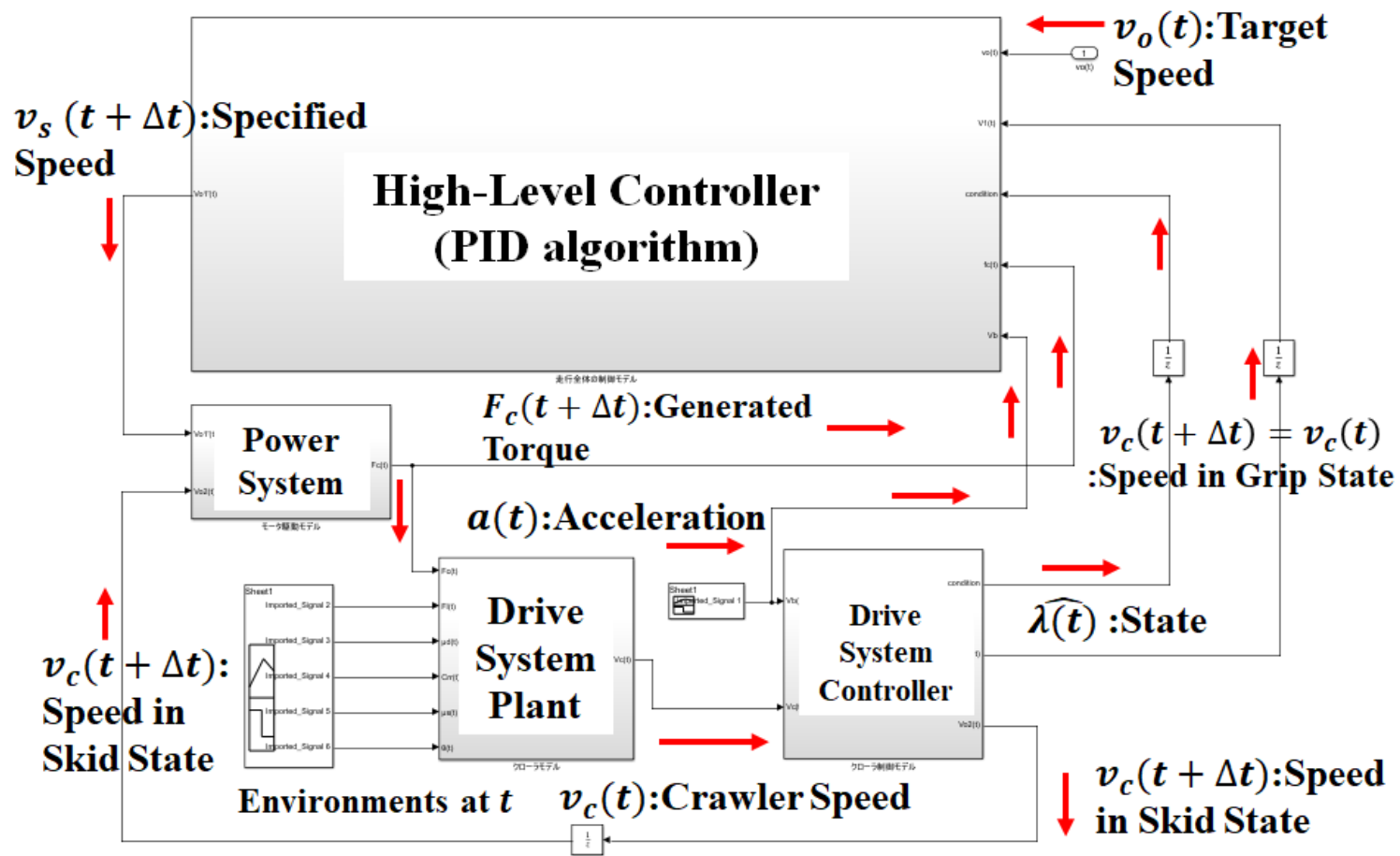

Figure 7. Top view of snow blower model

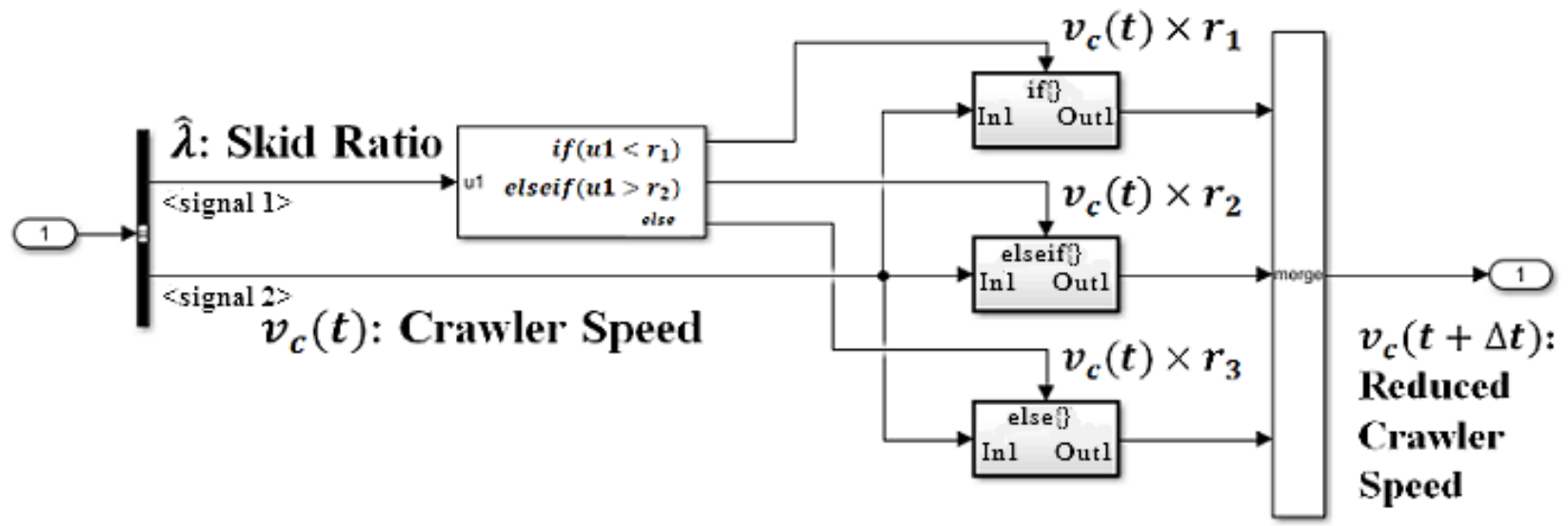

Figure 8. Skid suppression controller 
The skid suppression controller shown in Fig. 8 is included in "Drive System Controller" block. The current crawler velocity of snow blower $V_{c}(t)$ and the estimated skid ratio $\widehat{\lambda(t)}$ calculated by equation (5) from the acceleration $a(t)$ are inputs to this controller and the crawler speed in the next time step $V_{c}(t+\Delta t)$ is an output.

The parameters in Fig. 8 are shown in Table 1. Here, the skid suppression control is executed in three stages, however, any number of stages can be implemented. The actual values of parameters are shown in experimental results.

Table 1. Crawler Speed Reduction according to Skid Ratio

\begin{tabular}{|c|c|c|}
\hline No. & Skid Ratio & Reduced Speed \\
\hline 1 & $\lambda \widehat{(t)}<s_{1}$ & $V_{c}(t)=V_{c}(t+\Delta t) \times r_{1}$ \\
\hline 2 & $s_{1} \leq \lambda \widehat{(t)} \leq s_{2}$ & $V_{c}(t)=V_{c}(t+\Delta t) \times r_{2}$ \\
\hline 3 & $s_{2}<\lambda \widehat{(t)}$ & $V_{c}(t)=V_{c}(t+\Delta t) \times r_{3}$ \\
\hline
\end{tabular}

Here, the parameters $s_{i}(i=1,2$, and 3$)$ are such ones to divide the skid ratio into three stages and $r_{i}$ ( $i=$ 1,2 , and 3 ) are reduction ratios of crawler speed. As described in section 3.1, it is necessary to reduce the crawler speed to avoid stuck or lock state and to go back to the grip state. The drive system controller corresponds to a Virtual Twin which generates the next clawer speed $V_{c}(t+\Delta t)$ from the output of Digital Twin as shown in Fig. 9. The MBD method is used for "designing" controller systems while the Hybrid Twin is used for "operating" the completed controller system dynamically.

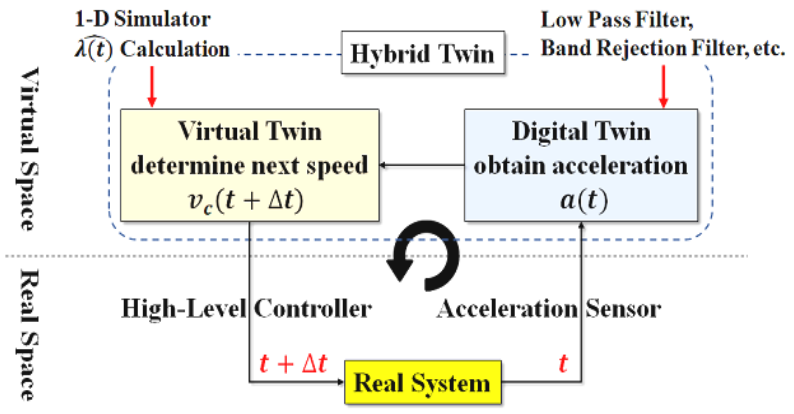

Figure 9. Actual Hybrid Twin with drive system controller

\section{Experimental Results}

\subsection{Snow Blower and Acceleration Sensor}

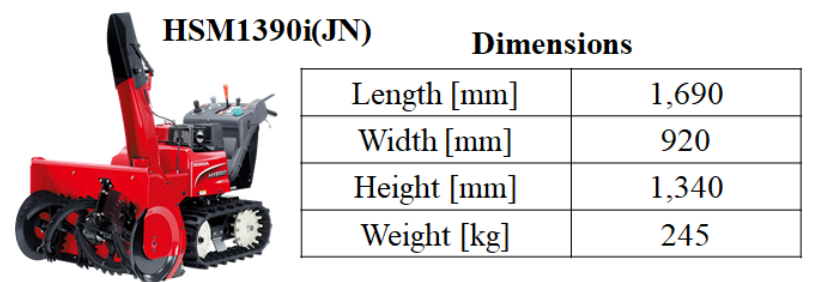

Figure 10. Snow blower used in experiments
The snow blower [12] taken as an example is shown in Fig. 10. This is the actual snow blower which can be obtained as a product.

As described in section 3.2, the skid ratio $\hat{\lambda}$ is estimated from the acceleration $a$ by using equation (5). The acceleration sensor which is incorporated in the snow blower is shown in Fig. 11. This 6-axis gyro sensor, "InvenSense MPU-9250" [13], is cost effective and it can be connected to the ECU in the snow blower by way of CAN (Control Area Network). This sensor corresponds to the IoT device which extracts the numerical data (Digital Twin) from the real system as shown in Fig. 9.

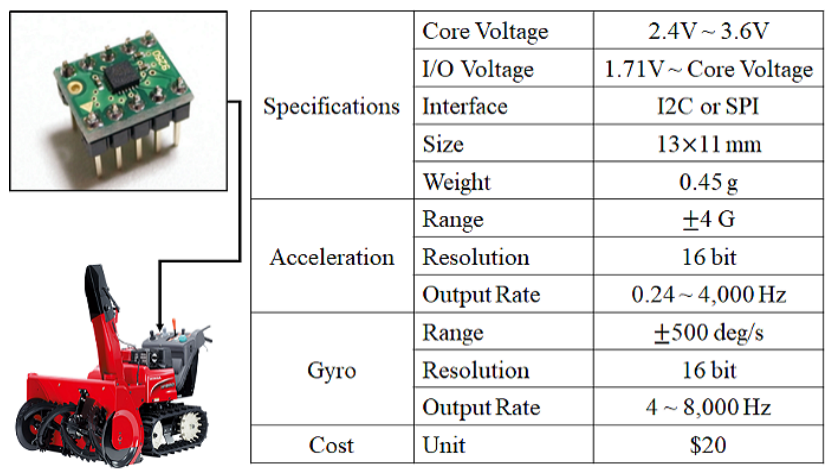

Figure 11. Specification of acceleration sensor

\subsection{Filtering of Acceleration Sensor Signals}

The filters for noise reduction of acceleration sensor signals are suggested and evaluated. Three kinds of filters are tested against the acceleration sensor signal obtained during driving on the flat asphalt floor. Here, two snow blowers are serially connected by a cable and the latter snow blower tows the former one to skid it as shown in Fig. 12.

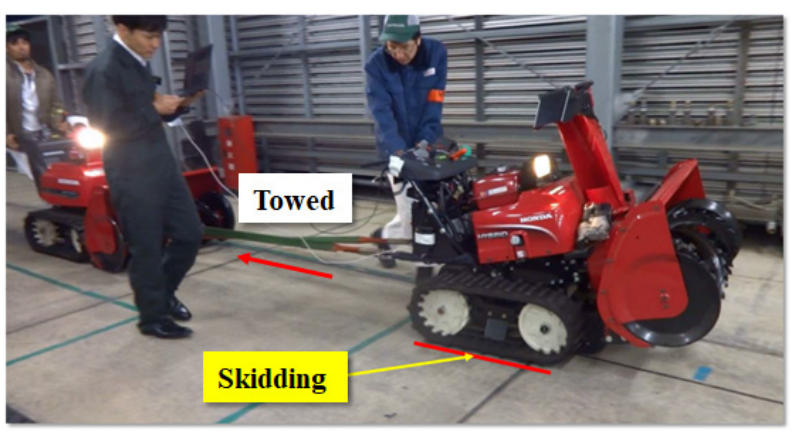

Figure 12. Skid state generation by towing

The raw signal obtained from the acceleration sensor has much noise because of vibrations of auger and engine as shown in Fig. 13. Here, the typical band rejection filter removing the noises ranging from $1 \mathrm{~Hz}$ to $1 \mathrm{MHz}$ is applied. Its transfer function is shown in equation (6).

$$
H(s)=\frac{s^{2}+\omega_{1} \omega_{2}}{s^{2}+\left(\omega_{1}+\omega_{2}\right) s+\omega_{1} \omega_{2}},
$$

where, $\omega_{1}=2 \pi \times 1$ and $\omega_{2}=2 \pi \times 10^{6}$. 


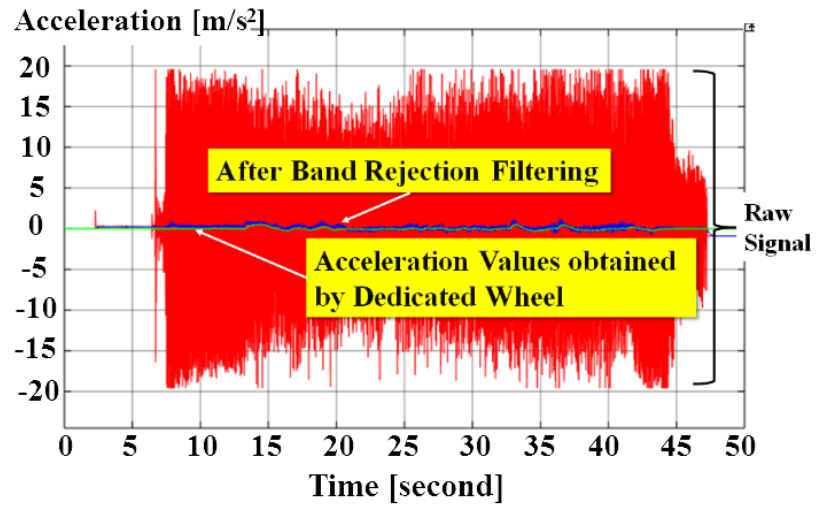

Figure 13. Acceleration signal after band rejection filtering

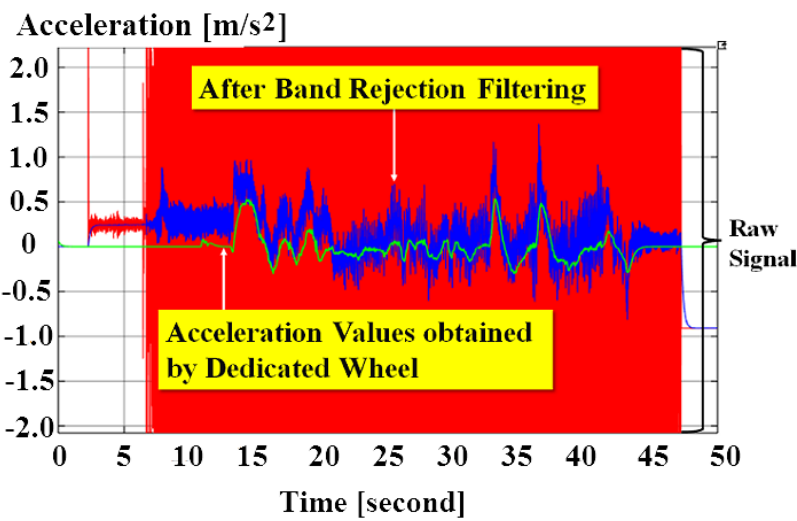

Figure 14. Acceleration signal after band rejection filtering (magnified)

The acceleration signals obtained by sensor after applying the above band rejection filter and by the dedicated wheel, respectively, are shown in Fig.'s 13 and 14. In these figures, the horizonal axis and the vertical axis mean the time and acceleration, respectively. The actual dedicated wheel measuring the speed is shown in Fig. 15 and the acceleration signal calculated from this wheel is set as a goal.

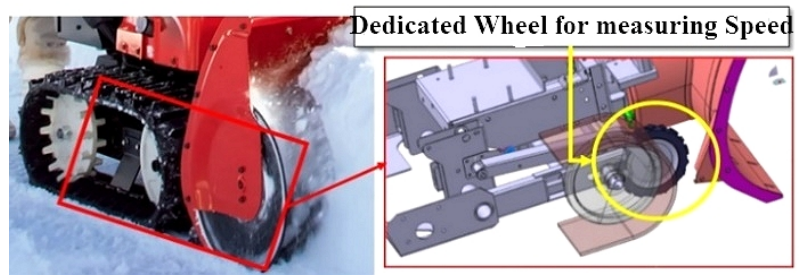

Figure 15. Dedicated wheel for measuring speed

As shown in Fig. 14, the signal after applying the band rejection filter still has large oscillation. Then, the low pass filter shown by equation (7) is further applied.

$$
H_{c}(z)=\frac{c z}{z+(c-1)}
$$

where, the cycle $T=0.004$ [sec] and $c=0.01$.
The result is shown in Fig. 16. The oscillation of signal obtained by the acceleration sensor is reduced and the signal's behavior is relatively the same as that obtained by dedicated wheel. However, from 0 to around 14 seconds when the snow blower is holding, the signal measured by acceleration sensor is not 0 while the signal obtained by dedicated wheel remains 0 .

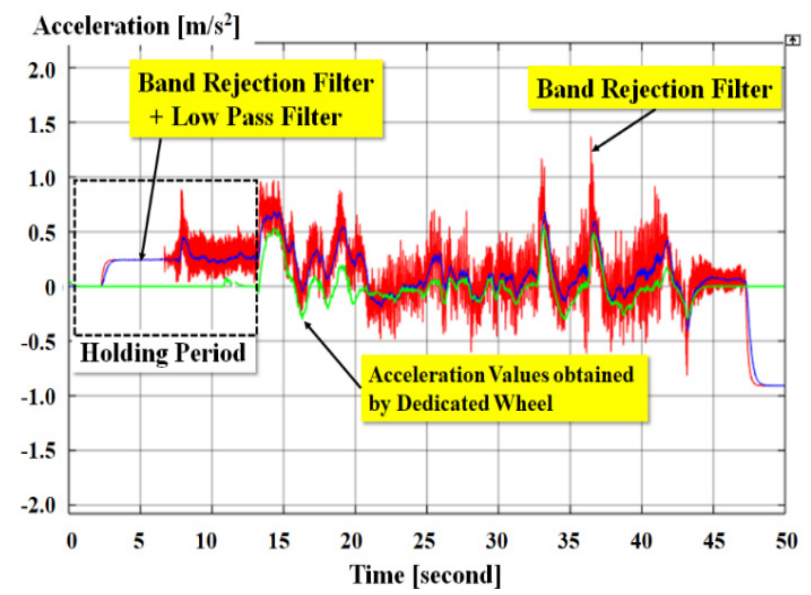

Figure 16. Acceleration signal obtained after low pass filter application

Then, the gravity component is tried to be removed from the signal because it is likely that the gravity component exerts some influence on the acceleration signal. The acceleration sensor has a clinometer and its measurement result can be used. The obtained signal is transformed by applying the filter shown by equations (8) and (9).

$$
\begin{aligned}
& \hat{A}_{x}=A_{x}-g \sin \hat{\theta}, \\
& \hat{\theta}=\tan ^{-1} \frac{A_{x}}{\sqrt{A^{2} y^{+A^{2} z}}},
\end{aligned}
$$

where, $\hat{A}_{x}, A_{x}, A_{y}, A_{z}, g$, and $\theta$ are estimated $x$-axis acceleration, the value of acceleration sensor in $x$-axis direction, the value of acceleration sensor in $y$-axis direction, the value of acceleration sensor in $z$-axis direction, gravity acceleration and gradient angle, respectively. The filtered results are shown in Fig. 17, and the oscillation of acceleration signal obtained by the acceleration sensor (shown as "After Gravity Component Rejection") is smaller than that obtained by dedicated wheel when the acceleration range is magnified. The behaviors between these signals are relatively similar with maximally $0.2\left[\mathrm{~m} / \mathrm{s}^{2}\right]$ error and removing the gravity component is effective for utilizing the acceleration signal from the sensor. On the other hand, the elimination of gravity component makes it difficult to obtain the precise acceleration value when the snow blower moves on the slope. 


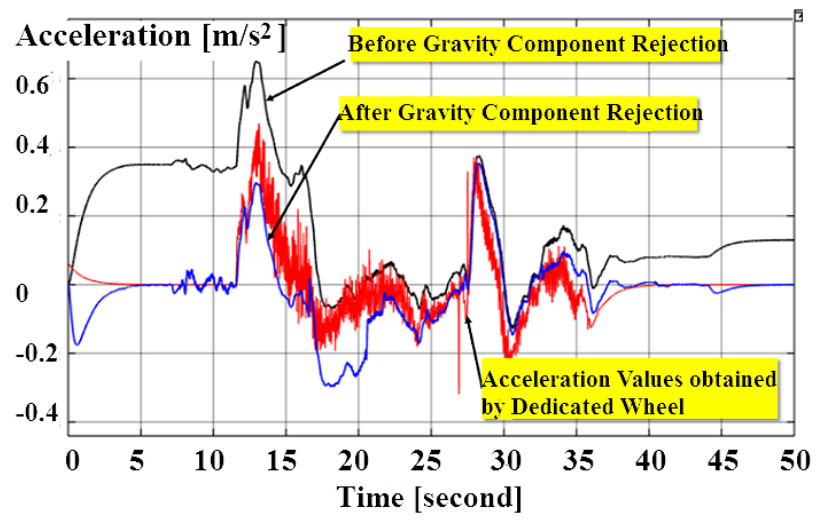

Figure 17. Acceleration signal after gravity component filtering

\subsection{Evaluation of Skid Suppression Control}

The skid state detection and its suppression control problems mentioned in section 2.1 are tried to solve by using the suggested method. The experiments shown in Table 2 are executed.

Table 2. Details of experiments

\begin{tabular}{|c|c|c|c|c|}
\hline No. & $\begin{array}{c}\text { Ground } \\
\text { Condition }\end{array}$ & $\begin{array}{c}\text { Driving } \\
\text { State }\end{array}$ & $\begin{array}{c}\text { Slope } \\
\text { (degrees) }\end{array}$ & $\begin{array}{c}\text { Maximum } \\
\text { Speed }(\mathrm{km} / \mathrm{h})\end{array}$ \\
\hline 1 & Snowy & $\begin{array}{c}\text { without } \\
\text { Skid }\end{array}$ & $4 \sim 8$ & $3(0.83 \mathrm{~m} / \mathrm{sec})$ \\
\hline 2 & Snowy & with Skid & $4 \sim 8$ & $3(0.83 \mathrm{~m} / \mathrm{sec})$ \\
\hline
\end{tabular}

In experiment No.1, the skid ratios obtained by acceleration sensor and dedicated wheel are shown in Fig. 18. The vertical axis is the skid ratio. In the comparison of estimated skid ratio with the one obtained by dedicated wheel, the suggested estimation method from the filtered acceleration sensor signal and equation (5) is seemed to be practical except in holding periods. In the holding period, the crawler speed $V_{c}$ is 0 and the skid ratio $\lambda$ cannot be defined, however, this exceptional process has not yet been implemented and the skid ratio is mistakenly 1 .

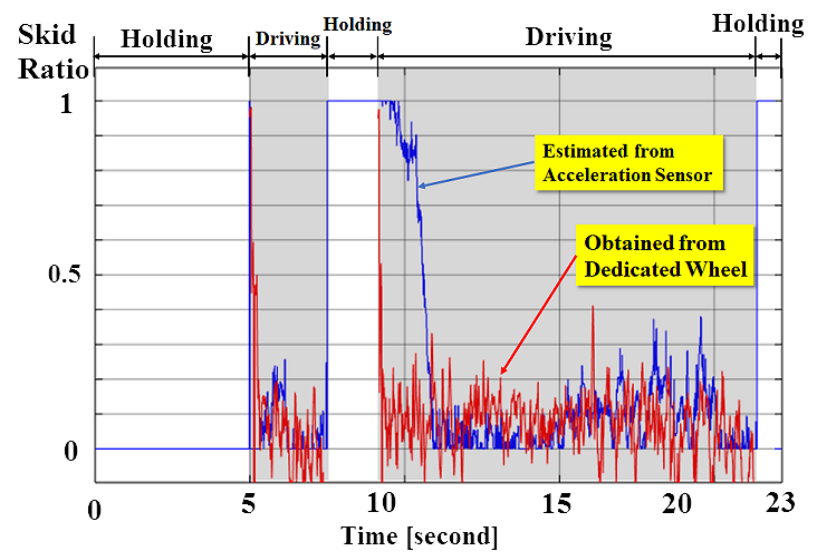

Figure 18. Comparison of skid ratios between estimated and measured

Moreover, when the snow blower is accelerated from just after holding period, the estimated skid ratio from sensor does not coincide with that obtained by dedicated wheel. This is because of the gravity component elimination and the acceleration or deceleration value cannot precisely obtain when the snow blower drives on a slope. This problem must be improved in future.

Next, in the experiment No.2, it is verified whether the estimated grip or skid state coincides with the one obtained by dedicated wheel. The result is shown in Fig. 19.

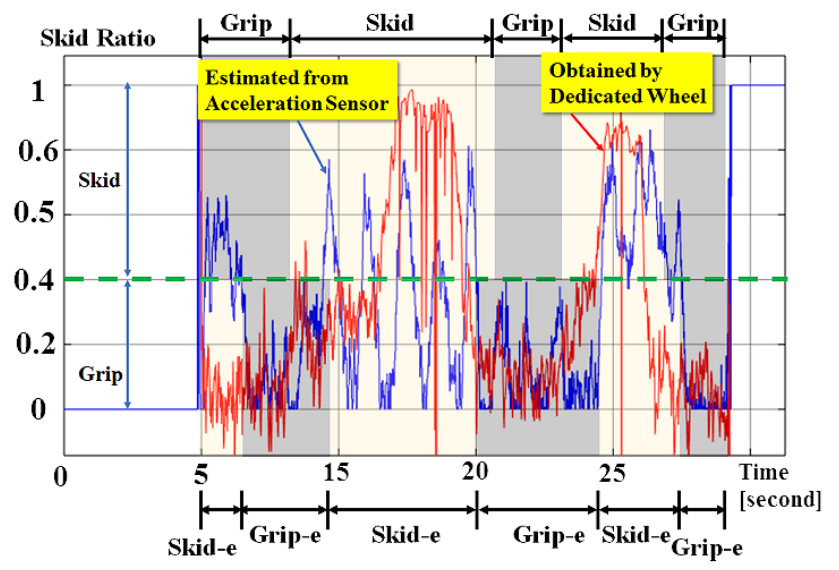

Figure 19. Comparison of skid and grip states

The driving states shown in the upper part of this figure mean the driving states of former snow blower and "Skid" state is generated when it is towed by the latter cable-connected snow blower in Fig. 12. The driving states shown in the lower part of this figure, such as "Grip-e" or "Skid-e" is the estimated ones by acceleration sensor based on the method described in section 5.2. The skid state, "Skid-e," is determined when the estimated skid ratio is greater than the specified threshold 0.4. Then, it is shown that there are some differences among time intervals of grip or skid states between generated and estimated cases with the maximum difference of 2 seconds. In the time interval from 5 to 7 seconds, the estimated skid ratio from acceleration sensor is large and the driving state in this time period is mistakenly estimated as "Skid-e." This may be from the same reason mentioned with respect to Fig. 18. In section 4.2, three-stage skid suppression is stated but here, two-stage skid suppression is seemed adequate because of the insufficient accuracy of skid ratio estimation. When the skid ratio accuracy is improved, three or more stage skid suppression may be implemented. The suggested two-stage skid suppression method is shown in Table 3.

Table 3. Two-stage skid suppression method

\begin{tabular}{|c|c|c|}
\hline No. & Skid Ratio & Reduced Speed \\
\hline 1 & $\lambda \widehat{(t)} \leq 0.4$ & $V_{c}(t)=V_{c}(t+\Delta t)$ \\
\hline 2 & $0.4<\lambda \widehat{(t)}$ & $V_{c}(t)=V_{c}(t+\Delta t) \times 0.5$ \\
\hline
\end{tabular}

The feedback path in the Hybrid Twin shown in Fig. 9 is scheduled to be evaluated in future. This is the verification whether the snow blower can go back to grip state within specified time interval when the two-stage skid suppression method applied. 


\section{Conclusions}

An IoT device utilization method in the framework of Hybrid Twin for work vehicle's control is suggested. Four such driving states as grip, skid, stuck and lock are defined. In autonomous driving, such state as stuck or lock must be avoided because the work vehicle in such states cannot escape by itself. The skid state is the entrance to these states and the speed reduction of driving device in skid state is a solution to get the work vehicle in skid state back to grip state. The skid ratio for quantifying the degree of skidding and the speed of driving device (e.g., crawler) is reduced based on the skid ratio by using three-stage skid suppression method. The Hybrid Twin is constructed with the skid controller as a Virtual Twin and with the acceleration sensor as a Digital Twin. The filtered acceleration signal obtained from real system is an input to the Virtual Twin and the speed of driving device is given to the real system as the specified speed in the next time step. The experimental results show that not the three-stage but the two-stage skid suppression method is efficient because of the insufficient noise reduction. However, the two-stage skid suppression method itself would have a promising performance in practical applications. Actually, the number of stages in skid suppression method should be flexible according to the performance of IoT device. As future work, the noise reduction should be improved and the skid suppression method should be implemented in a Hybrid Twin approach.

\section{Acknowledgements}

This is the research collaboration between Honda Motor Co., Itd. and Gunma University. The authors express their sincere appreciation for giving them the opportunity to research the controlling system for work vehicles and for the financial supports.

\section{REFERENCES}

[1] HONDA. CSE2019 Autonomous Work Vehicle, Online available from https://global.honda/innovation/CES/2019/a utonomous_work_vehicle.html
[2] Shigeki Inaba. The research for travel resistance of agricultural rubber crawler device caused by the rolling resistance of track rollers, Bull. Fac. Agr., Saga Univ., 88, pp.1-41, 2013

[3] Masashi Kawabata and Yoshimune Mori. Technology for Simulating Dynamic Motion in Hydraulic Excavators, Research and Development, Kobe Steel Engineering Reports, vol.62, No.1, pp.37-40, 2012

[4] MathWorks. Modeling an Anti-Lock Braking System, MathWorks, Online available from https://jp.mathworks.co $\mathrm{m} / \mathrm{help} /$ simulink/examples/modeling-an-anti-lock-braking-s ystem.html?lang=en.F

[5] Manfred Broy, Martin Feilkas, Markus Herrmannsdoerfer, Stefano Merenda and Daniel Ratiu. Seamless Model-Based Development: From Isolated Tools to Integrated Model Engineering Environments, Proceedings of the IEEE, vol.98, no.4, pp.526-545, 2010

[6] Yuki Shimamura, Yoichi Shiraishi, Kazuhiro Motegi, Yasuhiro Kobayashi, Takahiro Koga, Eiji Nakamoto, Jun Uchida and Takashi Todo. A Motor Modelling for Micro-Mobility by using Model Based Development method, Proceedings of 57 th Joint Conference on Automatic Control, 2A06-4, 2014

[7] Kazuhiro Motegi, Atsuhiro Aoki, Satoshi Sekine, Munetaka Nakata and Yoichi Shiraishi. A Proposal of General-Purpose Control Framework for Motorized Mobilities, Proceedings of the SICE Annual Conference, pp. 1632-1635, 2018

[8] Francisco Chinesta, Elias Cueto, Emmanuelle Abisset-Chavanne and Jean Louis Duval Virtual, Digital and Hybrid Twins: A New Paradigm in Data-Based Engineering and Engineered Data, Archives of Computational Methods in Engineering, DOI:10.1007/s11831-018-9301-4, 2018

[9] Abdulmotaleb El Saddik. Digital Twins: The Convergence of Multimedia Technologies, IEEE MultiMedia, vol.25, no.2, pp.87-92, 2018

[10] Kiyoshi Fujii and Hiroshi Fujimoto. Slip ratio control based on wheel speed control without detection vehicle speed for electric vehicle, IEE of Japan Technical Meeting Record, VT-07-24, pp.27-32, 2007

[11] Atsuhiro Aoki, Hideaki Shimamura, Munetaka Nakata, Kazuhiro Motegi, Yoichi Shiraishi. A New Skid Suppression Method for Avoiding Stuck State in Work Vehicle's Control, Proceedings of the SICE Annual Conference 2019, pp. 1594-1599, 2019

[12] HSM1390 (JN). Online available from https://www.honda.c o.jp/snow/lineup/medium-hybrid01/hsm1390i-jn.html

[13] MPU-9250. Online available from https://www.invensense. com/products/motion-tracking/9-axis/mpu-9250/ 